\title{
FDGRS: Improve Accuracy of Face Detection and Gender Recognition in Complex Lighting Environment by Image Enhancement Techniques
}

\author{
Reenu Rani \\ RTU, Kota \\ RCEW, Jaipur
}

\author{
Subhash Chandra Jat \\ RTU, Kota \\ Assistant Professor, RCEW, Jaipur
}

\begin{abstract}
This work focuses on the area of face processing and aims at designing a reliable framework to facilitate face, and gender recognition. Gender recognition aims at recognizing a person's gender (Male/Female). Automatic Gender recognition has become relevant to an increasing amount of applications gender recognition by face such as human computer interaction systems, content based image search, video surveillance, and more. Extensive experiment shows that the proposed model is able to capture both global and local information about faces. Tasks perform by system algorithm face detection, histogram of that particular for getting image information, reduce the image information by using histogram equalization and adaptive histogram equalization, noise remove by proposed NRCustom filter, human face characteristic extraction system and finally feature vector matching, classification. For experiment, purpose we adopted colored face image with different complex lighting condition (dark/dim/shaded) of random size and perform their simulation. Then finally, the system classifies the gender (Male/Female), count total no of images in database and separate number of males and females are present in the database.
\end{abstract}

\section{Keywords}

Pre-processing, feature extraction, Gender recognition system

\section{INTRODUCTION}

We are solving many real life problems with the help of computer vision techniques; the broad utilization of PC vision innovation frequently requires perceiving human attributes (e.g., age, gender and ethnicity) in a typical domain [3]. The field of face processing requires many aspects: analyse face structure and determine the exact location of face elements, such as eyes, noses, mouths, eyebrows, lips and cheeks. Another is the extraction of relevant information from these detected elements which can provide us with useful information regarding the identity, age, ethnicity and gender of a person.

Two separate problems are studied: face detection and the corresponding gender of face image apply on color images. Gender can be classified in two classes i.e. (M/F). Distinctive appearance of the face itself like, face image, skin shading and color, dark and shaded has a method of changeability prompts troubles in gender acknowledgment framework.

There are four main phases: apply pre-processing on image, feature extraction, classification and evaluation together develop a complete automatic gender recognition system. Successful gender acknowledgment is key and basic for some applications in the business areas, for example, human-PC communication and PC supported physiological or mental examination. Targeted advertisements, Surveillance System, Commercial Development, and Demographic Research.
Objective: The proposed work revolve around developing a FDGR System which is robust enough to accurately differentiate between genders by combining more than one aspects of gender recognition. The objective is decomposed into three smaller objectives which can be achieved separately. The first objective is to development an advanced algorithm to detect skin area under different illumination conditions (dim/dark/shaded) images, it may be adapted counter color and contrast imbalance of face images.

The second objective is to improve the accuracy of existing methods. General gender recognition system see Figure 1.

Organization: The paper is organized into the following sections. Section 2 deals with the literature survey of few related important papers work in utilized for the dissertation work. In Section 3 existing methods. In Section 4 description of the proposed approach methodology. In section 5 specified problems including all the solution and result analysis contains all the evaluation regarding proposed work it deals with MATLAB GUI block and guide. In section 6 conclusions and outcomes after applying the proposed approach on the images of the existing work as well as on new images.

\section{LITERATURE SURVEY}

There we have two problems face detection and feature extraction block, for the sake of completeness for Gender Discrimination. This section addresses recent developments in various related methods of gender recognition by face images using image processing. Thus, the literature provides a systematic review of the Gender Discrimination related problems.

Each person has distinctive face, meaning that each face looked unlike. Face detection approach divided into four categories: information based, include invariant, layout coordinating and appearance situated in past work [7]. The cascaded face detector by Viola and Jones (2001) is exceptionally notable appearance based technique [5]. The detector searches face starting from left top corner to bottom right corner.

Existing initial overviews for algorithms identified with gender orientation estimation. Based on these works we can presume that gender orientation estimation remains a testing errand, which is intrinsically connected with various biometric modalities [6] including fingerprint, face, iris, voice, body shape, gait, signature, DNA, as well as clothing, hair, jewellery and even body temperature.

Previous works focused on finding good discriminative features for classification. Most of the past techniques utilize at least one blend of features, for example, LBP, SURF, HOG or SIFT. These methods based on geometric, appearance based features and joining both features at same time of the image. 
We extensively arrange extraction techniques for confront based gender orientation characterization into geometric-based and appearance-based techniques, following. The previous work depends on estimations of facial points of interest.

In this paper Mahmoud Afifi et al. [1] proposed a method which is based on facial gender recognition rather than managing the face image as an individual component, they depend on the mix of isolated facial features and an all-encompassing element which they call the foggy face. At that point, they utilize these features to prepare profound convolutional neural systems taken after by an AdaBoost-based score combination to construe the last gender orientation class. They evaluate their method on four challenging datasets to demonstrate its efficacy in achieving better or on-par accuracy $88.09 \%$ with state-of-the-art methods. In addition, they present another face dataset that strengthens the difficulties of impeded appearances and enlightenment changes, which we accept to be a truly necessary asset for gender orientation order examine.

In this paper [2] an exceptionally mainstream technique in view of the idea of decreased dimensionality is Principal Component Analysis (PCA). PCA is a system in view of the idea of eigen faces and was first presented by Kirby and Sirivich in 1988 Eigen faces have been utilized to track human faces. It is one of the more successful techniques of face recognition and easy to understand and described using mathematics [5]. They utilize principal component analysis way to deal with store an arrangement of known examples in a reduced subspace representation of the image space, where the Eigen vectors of the preparation image set range the subspace.

This approach makes use of skin color to isolate the face. Any non-skin color region within the face is viewed as a candidate for eyes or mouth [6]. Color based feature extraction use color models such as RGB, YCbCr or HSV with certain range of color pixels, skin region is detected [9].

In view of this [9], they proposed a compact and efficient LBP feature extraction method which learns discriminative LBP histogram bins using Adaboost. Based on comparison with pixel intensity based global method, they showed performance improvement using boosted LBP features. Further, it relies only on the first-order derivative in local neighbour and discards directional information which may limit discriminative information.

A.R. Ardakany et al. [4] proposed a novel method of feature extraction to gender classification, which extracts geometric, based and appearance based features at the same time. This feature extraction performed by computing the derivative in all pixels of face images and then constructing a histogram based on edges magnitudes and directions. For experiment purpose, they were used FERET database. Classifiers such as LDA, Adaboost and SVM with linear kernel. There for increase the performance of SVM first linear kernel used than they found that using SVM with linear kernel give best result and accuracy by proposed method. In this work histogram based on magnitude \& direction of the edge while an existing algorithm, define histogram based on intensity.

Yujie Dong et al. [12] investigate gender recognition method based on the shape of eye and eyebrow, because the shape of eyes and eyebrow large difference in the structure and variety that makes each human face very much different. They then extracted nineteen shape-based features from each eyebrow. Each feature was normalized to scale [l $\left.\begin{array}{ll}0 & 1\end{array}\right]$ based on min-max normalization, and compared three different classification methods: Minimum Distance Classifier (MD),Linear Discriminate Analysis Classifier
(LDA) and Support Vector Machine Classifier (SVM).with two datasets, preliminary study demonstrates the feasibility of utilizing shape-based features extracted from non-ideal images for biometric recognition and gender classification.

By going through the papers in detail, we have found that most of the experiments performed in gray scale and colored images [1-12] reveal color information improves on face recognition and gender retrieval performance. In paper, geometric based methods, number of fiducially points considered to determine different gender. However, it was found that the geometric based methods are more complicated than appearance based methods in terms of implementing for Gender Recognition system because in geometric based methods feature points are need to be set manually. Most of the classifiers used are SVM, Adaboost and neural network.

\section{EXISTING METHODS}

There are two different methodologies, namely the 'Geometricbased' and the 'Appearance-based' parameterizations, which can be used in both of these approaches. These feature extraction techniques apply when we detect a particular face from the image, so the face detection task of locating the position and size of a face in a given input image. The most widely used algorithm for this task is by Viola and Jones [11].

After the detection of the face we use feature extraction. There are so many feature extraction methods used in gender recognition. In this section, only the most popular ones are presented: Intensity values, Haar-like features, Gabor Wavelets, Local Binary Patterns, Principle component analysis. After the feature extraction classification process is performed on extracted features there are most popular classifiers are SVM and Adaboost. The systems use standard dataset like FERET and CAS-PEAL face database.

\section{PROPOSED APPROACH METHODOLOGY}

Proposed work on Gender Recognition systems has studied and reviewed, state of the current technology summarized in the previous chapters, results of which have guided us to design a Gender Recognition system for future use.

A through survey has revealed that various methods and combination of these methods can be applied on face for gender recognition in both controlled and uncontrolled situations. Problem arises in uncontrolled condition when there are high rate of noises, lack of illumination etc. To mitigate the problems, we have proposed an approach where the first strategy is based on registering the input images prior to their classification.

\subsection{Proposed Algorithm}

In our proposed work, firstly we store live database of dark/ dim/ shaded face images.

The database images need of pre-processing so enhance these images by Histogram Equalization, Adaptive Histogram Equalization and face area detection. Secondly facial components were detected from selected image by using skin color segmentation and morphological operations. After extraction, the extracted facial components were subjected to proposed algorithm and give classification result.

Require: Test face image from face image database.

Ensure: Feature vector of input image $\left(I M_{\text {in }}\right)$

Output: Result of classification $(\mathrm{M} / \mathrm{F})$, total no of images in database, separate calculation of male and female images in database. 
Step-1: One pair of Maximum and Minimum points: For an $\mathrm{M}$ x $\mathrm{N}$ color image to gray scale, Every pixel shows grayscale value $x \in[0,255]$.

$$
\begin{aligned}
& \text { If } I M_{i n} \text { is colored then } \\
& G I M_{\text {in }} \leftarrow \text { Convert } I M_{\text {in }} \text { gray } \\
& \text { else } \\
& G I M_{\text {in }} \leftarrow I M_{\text {in }}
\end{aligned}
$$

Step-2: In the histogram $\mathrm{H}\left(I M_{\text {in }}\right)$, locate the maximum point $h(a), a \in[0,255]$ and the minimum point zero $\mathrm{h}(\mathrm{b}), \mathrm{b} \in[0,255]$.

$\mathrm{H}\left(G I M_{\text {in }}\right) \leftarrow$ produce Histogram of image $\left(G I M_{\text {in }}\right)$

$\mathrm{HE}\left(G I M_{i n}\right) \leftarrow$ produce Histogram Equalization of image $\left(G I M_{\text {in }}\right)$

Step 3: $\quad$ Save image and apply some other pre-processing on enhanced image.

It consist of Color conversion, Noise reduction, cropping skin area and resizing the image.

Step 4: $\quad$ Crop face image to convert it into standard face image.

Step 5: $\quad$ Extract $\mathrm{N}$ feature vector from image feature viz., total number of white pixels, and distance calculation, color moment.

Step 6: $\quad$ Matching distance of each class of the images in the database is calculated. The class having the minimum distance, the test image belongs to that class.

Step 7: $\quad$ Repeat step 1 to 6 for query images

\subsection{Proposed Model}

Gender recognition system model is see Figure 1.

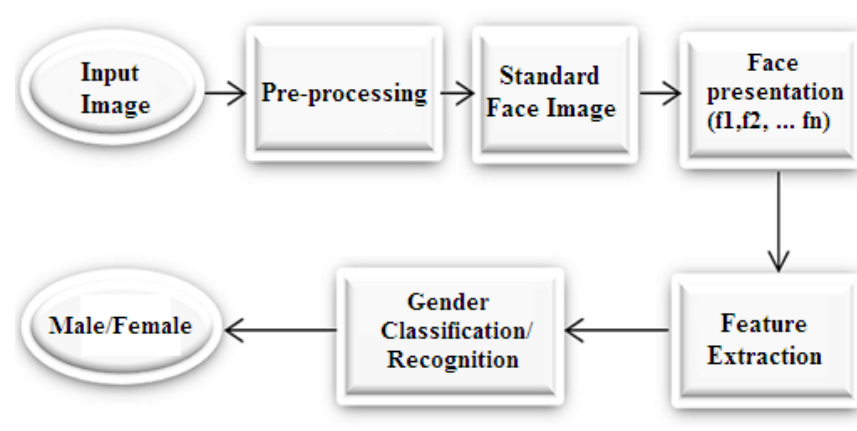

Fig. 1 Work flow of proposed approach

4.2.1 Training and Testing : We collected some male and female faces in our web database, for testing purpose we randomly selected one fifth face images from each categories and rest of the images are used as training images. We repeated this process many times for each class. We have taken static colored face images. We have tested proposed system on the images of different categories such as Images with little head bend; images under different lightning conditions \& background and with different image formats such as PNG, JPEG etc. However, challenges of process an image are illumination effect, pose, and image quality

The processing starts with load database and getting an input image from the database. The face image see Figure 10 is taken from web. So we have need apply some pre-processing on database images.

4.2.2 Input Image: First select an image this will go through face detection process. Whose main aim is to obtain different shape and size? We have used Dark/Dim face containing static color images for gender recognition system.

These D/D web face images have requirement of contrast improvement so we use two techniques such as Histogram Equalization and Adaptive Histogram Equalization. After this process all stored images required some other operation like edge detection, skin color segmentation, and morphological operation to detect only face skin area from the input image. For better classification results, the input images are generally preprocessed to reduce the effects of illumination, filtering, face area detection is a commonly used technique for this purpose.

4.2.3 Pre-processing: It includes a reduction in the size of the image to lower the computational burden. Pre-processing of an input face image will experience a few procedures keeping in mind the end goal to accomplish a changed face image.

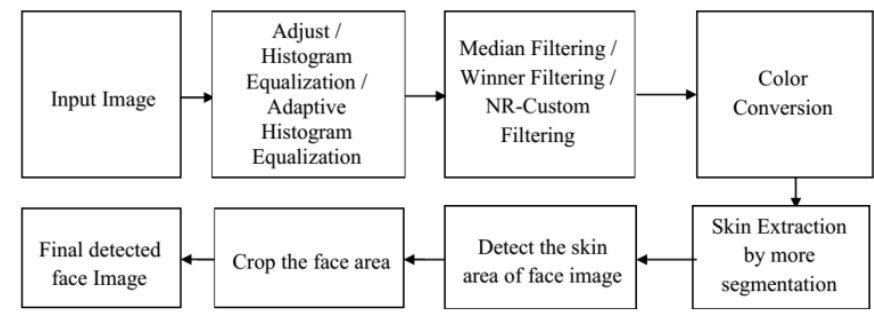

Fig.2 Steps of pre-processing

\subsubsection{Image Contrast Enhancement}

Several properties, for example, line, corners, spots, edges can be utilized as features for classifying face image. To obviously imagine the properties, a few image improvement system can be used. Complexity improvement is a procedure by which the pixel force of the image is changed to use the most extreme conceivable containers (Gupta et al, 2014). It very well may be ordered into two classes: (1) Local and (2) Global difference improvement.

\section{Global Contrast Enhancement}

Histogram Equalization: The histogram is a graphical representation of an image which demonstrates the recurrence of happening of information in the entire informational index. It plots the quantity of pixels for each tonal value. Consider an image with $\mathrm{G}$ add up to conceivable force levels. At that point, the histogram of the image in $[0, \mathrm{G}-1]$ is characterized as a discrete capacity:

$$
P\left(r_{k}\right)=\frac{n_{k}}{n}
$$

Where, $\mathrm{rk}$ is the $\mathrm{k}$ th intensity level in the interval. $\mathrm{nk}$ is the number of pixels in the image whose intensity level is rk. $\mathrm{n}$ is the total number of pixels in the image.

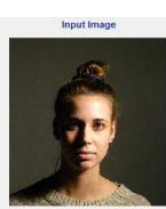

(a)

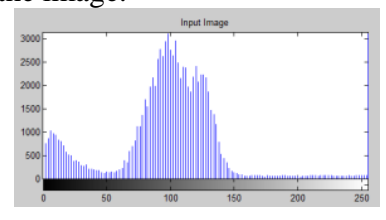

(b) 


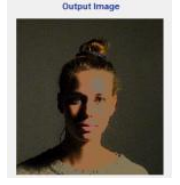

(c)

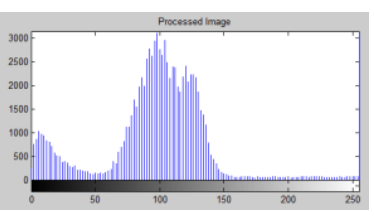

(d)
Fig. 3 (a) Original image (b) The histogram of the original image (c) Image obtain after HE (d) The histogram after enhancement (HE)

\section{B. Local Contrast Management}

Adaptive Histogram Equalization (AHE): it is unique in relation to conventional histogram equalization in it isn't global and it registers numerous histograms comparing to various segments of an image (Gupta et al, 2014). In this way, it is possible to improve the neighborhood differentiation of an image through AHE [7]. With $\wedge$ UL the doto of nower scopes of an image can be seen at t... the issue of numerous standard gadgets which can't delineate the full unique force run. Here, initial, a relevant area is characterized for each pixel in the image. The relevant district is the area focused about that specific pixel. This outcome in the neighborhood appropriation of intensities and last upgrading depend on neighborhood than the whole global area of the image.

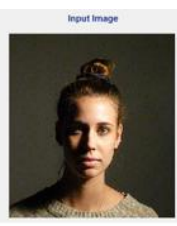

(a)

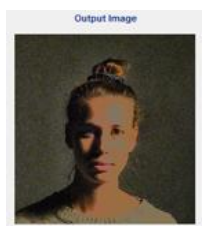

(c)

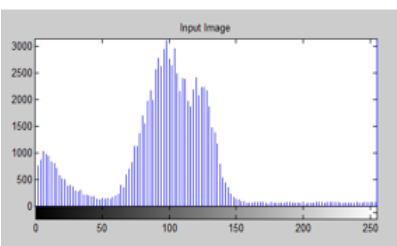

(b)

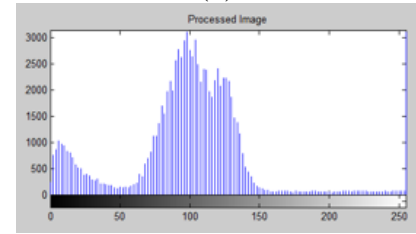

(d)
Fig. 4 (a) Original image (b) The histogram of the original image (c) Image obtain after AHE (d) The histogram after enhancement (AHE)

4.2.4 Noise Reduction: The process removal of noise called noise reduction. Noise is because of either some disturbance influence in system of electronic gadgets or due some issue in calculation. May in like manner be caused by consistent sources like particles of residue inside the camera and overheated or from defective CCD segments. For noise removal, some filters like linear, nonlinear, adaptive, filtering etc. are used. Here NRCustom proposed filter is used for noise removal.

\subsubsection{Noise Removal Proposed Filter (NRCustom)}

Therefore, our designed custom filter presents a technique based on pre-treatment of image which improves the quality of normal/dark/dim/shaded digital images. As we go into dark environment, the color starts to disappear according to their wavelengths. For example, firstly red color will disappear, secondly the green color. Also blue color falls in shortest wavelength category. The key goal of the algorithm is to improve the accuracy of dark and blurred digital images enhancement method / technology.
To solve the existing problems of image enhancement technology based on CLAHE and $\mathrm{L} * \mathrm{~A} * \mathrm{~B}$, we can use color space method. To overcome CLAHE output in the uneven lighting problems, the image has been removed by using image enhancement gradientbased smoothing. Put forward the key ideas of technology to improve the accuracy of the different low contrast image enhancement method. As degradation of images occur due to darkness and motion blur. These are the two main challenges posed to capture image. Non rigid deformation of scene is also sometime obtained due to space varying refraction.

Image denoising is likewise an important strategy. Image denoising model promotes the estimation of noise while preserving the edges. Gaussian filter is utilized to tackle thermal condition and info information is the noise image. Following technique is an attempt to reestablish the image distorted because of movement blur and noise issues.
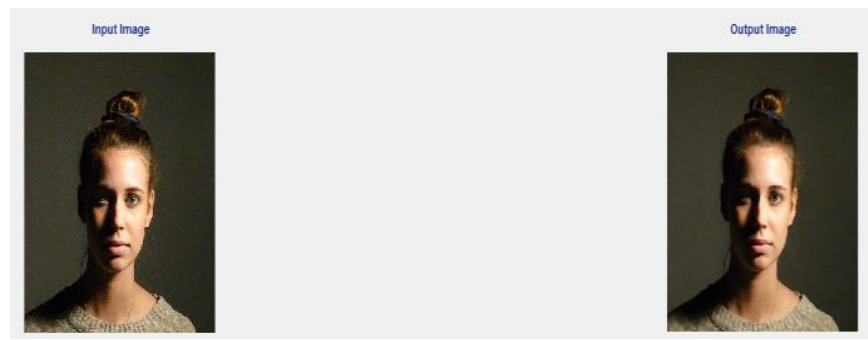

Fig.5 Filtered image by using NR-Custom filter

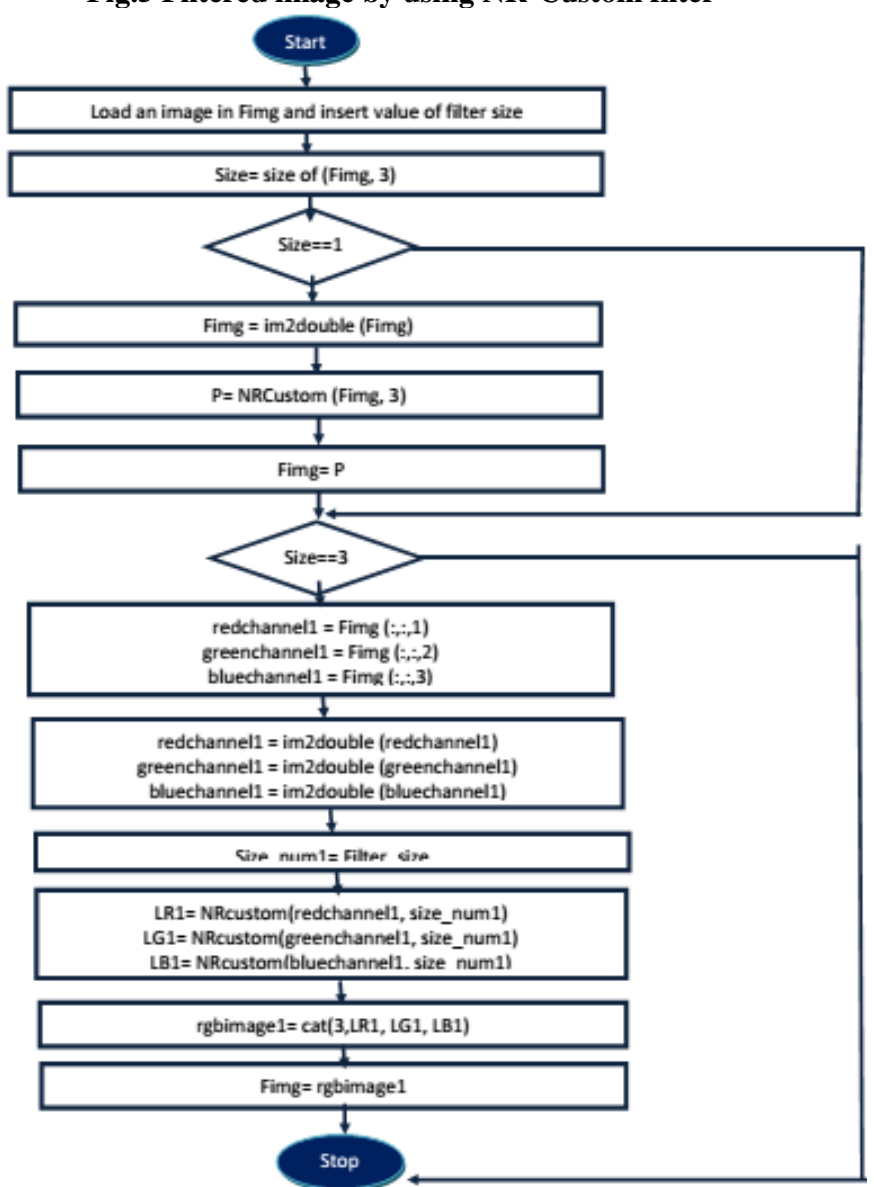

Fig. 6 Flow chart demonstrates the custom designed filter.

4.2.5 Color Conversion 
A RGB color image is an $\mathrm{MxNx} 3$ collection of color pixels relating to the red, green and blue segments that indicate a particular spatial area over entire image. RGB image have millions of color $[16,777,216] \mathrm{I}$ a single image [10]. RGB image is converted into gray image which have 256 colors [0-255] and binary image which have only two colors in image. This approach gives good results if arise an occurrence of gray scale images. Gray scale image comprises of force values between 0 to 255 , which needs to change over into 0 and 1 just as per their suitable class. Framework takes input image in gray tone (0-255) and utilizing a thresholding approach changes over them into two-tone images ( 0 and 1$)$, darkest pixels having the esteem 0 's compare to protest and white pixels having esteem 1's relate to foundation.

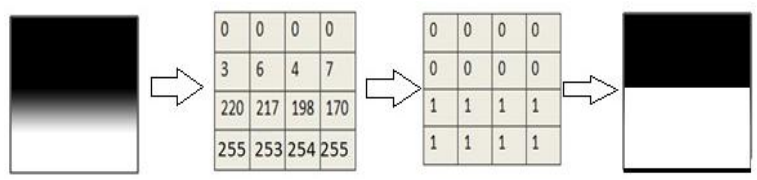

Fig.7 Gray Scale image go through thresholding and Binarization process

4.2.6 Edge Detection: It is mainly used for detecting and extracting the boundary features of image. The location has done at where the image brilliance changes forcefully and at where there will be discontinuities. The principle reason of recognizing sharp edges of images is to catch and watch where there is a change at a specific point, which can change the whole occurrence. In a perfect case, the result of edge detector may prompt to the development of set of associated curves that relates to variations in surface introduction.

Consequently, actualizing edge recognition calculation on any image may fundamentally decrease the amount of information to be prepared and furthermore removes the unfortunate information. There we use canny edge operator. Canny edge detector was developed by John F. Canny in 1986. It is used as multi-arrange calculation to identify an extensive variety of edges in images [10]. The nearby angle is given by Equations

$$
\begin{gathered}
G=\sqrt{G_{x}}{ }^{2}+G_{y}{ }^{2} \\
G=G_{x}+G_{y} \\
\theta=\arctan \left(\frac{G_{x}}{G_{y}}\right)
\end{gathered}
$$

Gradient along $\mathrm{x}$ direction and $\mathrm{y}$ direction is calculated by both equations as [10]:

$$
\begin{aligned}
& G_{x}=f(x+1, y)-f(x, y) \\
& G_{y}=f(x, y+1)-f(x, y)
\end{aligned}
$$
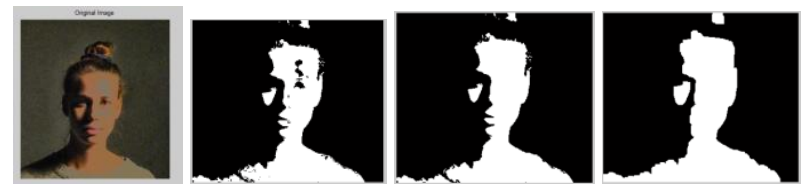

Fig. 8 (a) Original images, (b) Edge detection of input Image (c) Apply morphological operation (d) fill black holes

Image segmentation can be defined as dividing an image into different groups or partitions based on some homogeneity criteria like color, intensity, or texture etc.( Gonzalez et al, 2014) ) (Figure 8) demonstrates that skin color division completed a great job of dismissing non-skin colors from the input image. After that morphological opening task is performed to remove little protests from the image while preserving the shape and size of larger object in the image. The meaning of a morphological opening of an image is identification of edge taken after by dilation, utilizing the organizing components. Using 'imfill' function holes are also dilated. These holes are also filled see Figure 8(d). The considered skin region is then labeled. Bounding box is applied on each segment with the quantified window size, which is selected to meet the size of the face.

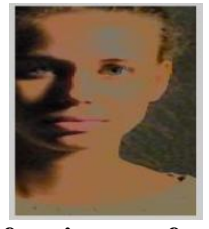

Fig. 9 Standard face image after pre-processing

4.2.7 Feature Representation and Extraction: It acquires relevant data representation to extract discriminative information. There can be an underlying structure that further describes differences between male and female faces better. Thus the feature detection module should be employed here. Templates created from extracted features are stored in the system dataset during the training phase for the purpose of matching when the test sample is presented during classification phase.

\subsection{Feature vectors}

Form feature vector is a one-dimensional portrayal of the shape, which acquired by applying the 8-point availability system on the form image. As first, we have to discover the centroid of facial objects. After this the feature vector representing the central distance between a point on contour (x,y) and the centroid (xc, yc) is given by

$$
F V c=(x-x c, y-y c, 0)
$$

Edge directions are used to describe the distribution of the edge points in each direction; it is calculated by counting the number of the pixels in each direction. Every component of face image has edges and with their direction, we calculate a feature vector for gender discrimination. The principle is that the image is processed in order to extract the edges and their direction. For this technique, we used Canny's operator for edge direction. Main advantage of selecting this feature is that the use of edge directions captures the general shape information and use of the histogram makes a method invariant to translation in the image. Scale invariance can be acquired by normalizing the histogram as for the quantity of edge focuses in the image. In addition, due to the color distribution information mainly concentrated in the loworder moment. So using only colors the first moment (mean), second moment (variance) and third moment (skewness) is sufficient to express the color distribution of an image. Compared with histogram, another advantage of this approach is that the features do not need to be quantified. Three low- order moments of colors expressed mathematically as:

$$
\begin{gathered}
\mu_{i}=\frac{1}{N} \sum_{j=i}^{N} p_{i j} \\
\sigma_{i}=\left(\frac{1}{N} \sum_{j=i}^{N}\left(p_{i j}-\mu_{i}\right)^{2}\right)^{\frac{1}{2}} \\
S_{i}=\left(\frac{1}{N} \sum_{j=i}^{N}\left(p_{i j}-\mu_{i}\right)^{3}\right)^{\frac{1}{3}}
\end{gathered}
$$

Here $P_{i j}$ is the image of the $j^{t h}$ pixel in the $i^{t h}$ color component. Thus, the total moment of color image has only nine components.

\subsection{Matching and Classification}


After extracting all the necessary features, the final task is to decide whether these features represent female or male face. It involves comparison of features extracted from the test sample with templates stored in the system dataset. Based on scores obtained from the comparison, the output of pattern classification block indicates the estimated class of the input test image.

The similarity between the query image feature vectors and feature vectors in dataset computed using the Euclidean distance formula.

$$
\operatorname{Sim}_{F V}(D)=\sqrt{\sum_{n=1}^{n=m}\left(S I_{n}-I D S_{n}\right)^{2}}
$$

SI represents the selected image and the IDS is the image dataset, $\mathrm{FV}$ is the feature vectors.

\section{Evaluation Protocol and Parameter Settings}

For the implementation of gender recognition system, the experimental setup includes both hardware and software requirements. The proposed work is implemented with MATLAB tool on Windows platform using operating system Window-7. We train our system before execution using the entire training image, which includes different format as well as different resolution Figure 10. Samples face images.

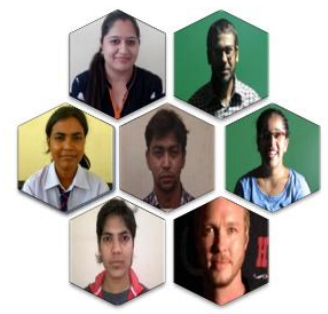

Fig.10 Sample Database images (Male/Female)

\subsection{User Interface with system}

At very first step in the processing of image indexing, using MATLAB 2013a open command window > start with run a main file is saved with the name "Gui", The first screen which appears after the command as see the Figure 11 which has three basic functionality, first one to set the input image and the second one is to apply pre-processing operation for getting standard image and third classify gender.

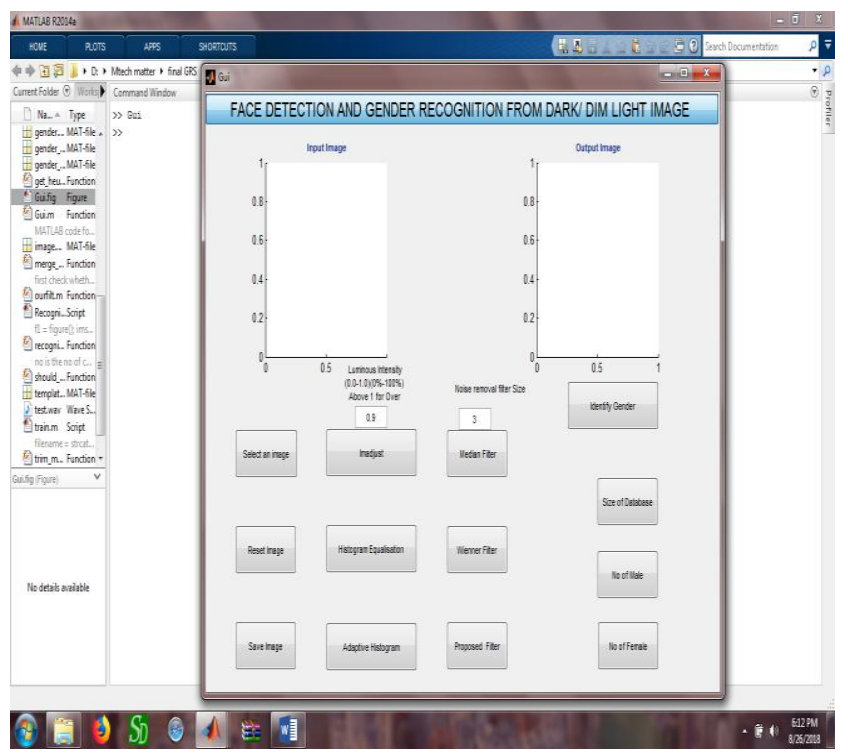

\section{Fig.11 GUI for Gender Identification System}

Upload an image over the system by clicking on button "Select an image" image display from database in axis handler axis 1 shown below in Figure 12.

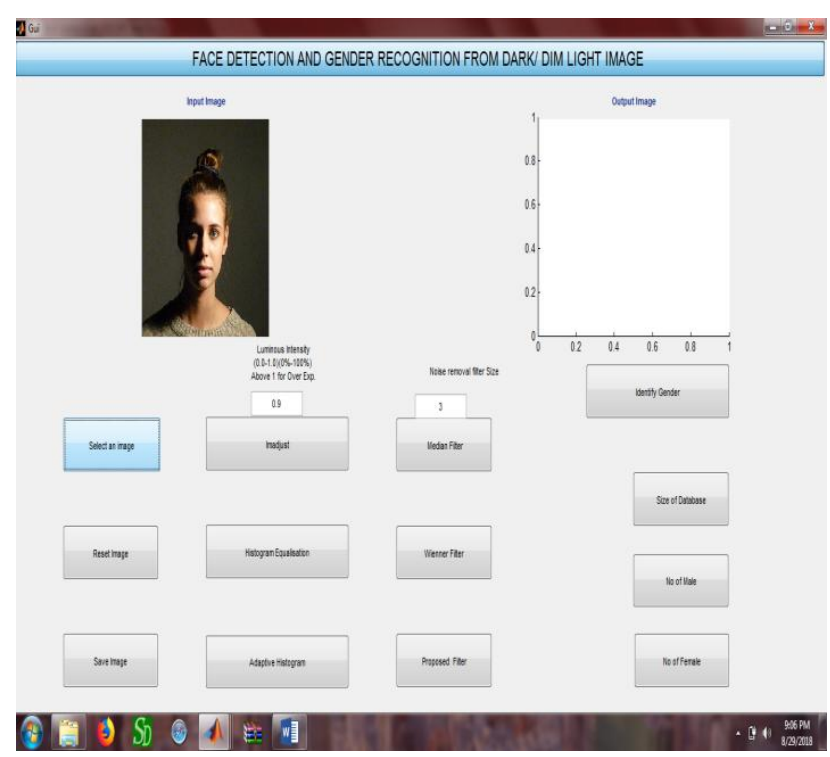

Fig.12 Screen showing selected image

After selecting the test image we can apply different operation according to image requirement, after the processing image see axis handler axis2 in Figure 13. We apply adaptive histogram equalization. We can reset or restore that particular image and we can apply another operation on same image this concept represent the reset by using reset button.

The save button represent image saving process, after the image pre-processing the standard image save in database and we can apply gender recognition on same image. Image contrast enhancements by using button imadjust, histogram equalization and adaptive histogram equalization button. After that we apply image filters on image if these have requirement. Results of classification see Figure 13, Figure 14.

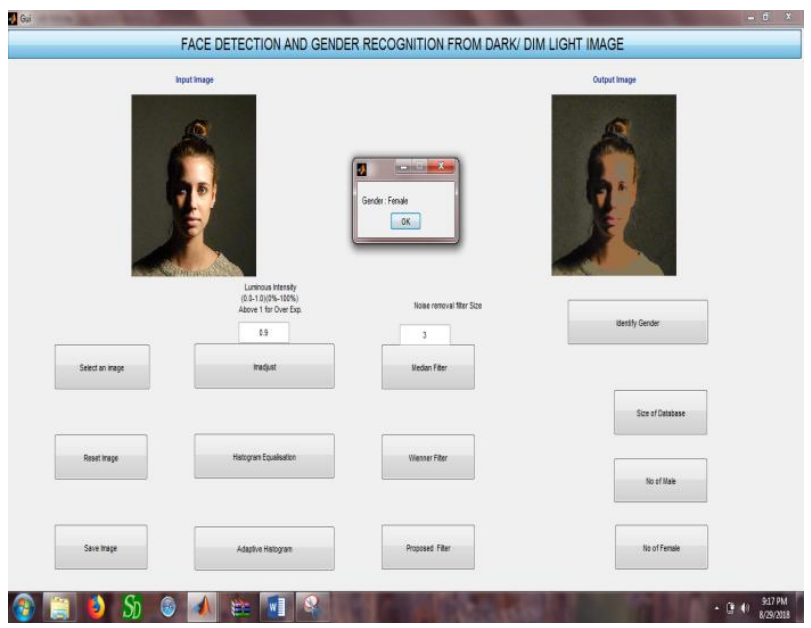

Fig. 13 Screen showing classification result 


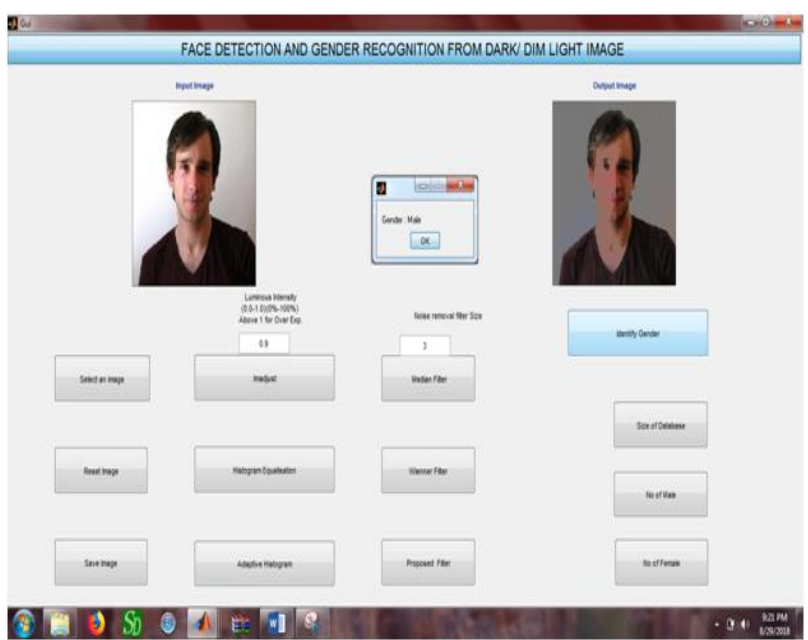

Fig. 14 Another case for classification

\section{Database Calculations}

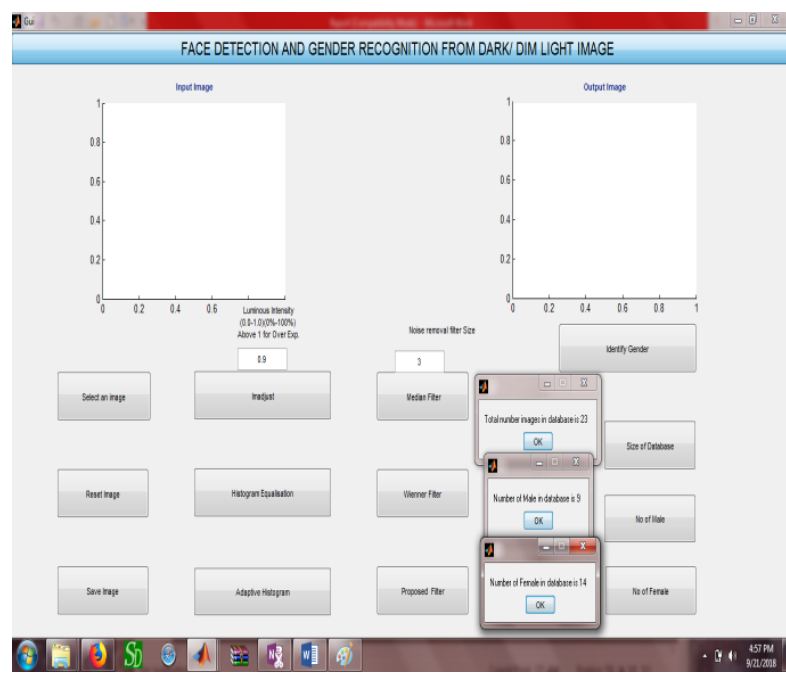

Fig. 15 Database calculations

5.2 Overall System Analysis: To verify the effectiveness of our approach, a FDGR System is performed over the entire system module. All the test images are basically divided into two classes. The overall system performance calculated based on these two classes.

Male accuracy is defined as the number of correctly classified male images, TM divided by the total number of male images $\mathrm{M}$. i.e.

$$
\text { Accuracy of male }=\frac{T M}{M}
$$

Female accuracy is defined as the number of correctly classified female images, TF divided by the total number of female images, F. i.e.

$$
\text { Accuracy of male }=\frac{T F}{F}
$$

Overall accuracy is defined as the sum of correctly classified male and female images divided by total number of images

$$
\text { Overall accuracy }=\frac{T M+T F}{M+F}
$$

\subsection{Comparison of proposed work}

\begin{tabular}{|c|c|c|c|}
\hline Sr. No. & Parameters & Previous work & Proposed work \\
\hline 1 & Title & $\begin{array}{l}\text { Deep Gender } \\
\text { recognition } \\
\text { based on } \\
\text { AdaBoost-based } \\
\text { Fusion of } \\
\text { Isolated Facial } \\
\text { Features and } \\
\text { Foggy Faces }\end{array}$ & $\begin{array}{l}\text { FDGRS: Improve } \\
\text { Accuracy of Face } \\
\text { Detection and } \\
\text { Gender Recognition } \\
\text { in Complex Lighting } \\
\text { Environment by } \\
\text { image enhancement } \\
\text { techniques }\end{array}$ \\
\hline 2 & $\begin{array}{l}\text { Operation } \\
\text { performed }\end{array}$ & $\begin{array}{l}\text { Lighting } \\
\text { invariant } \\
\text { enhancement } \\
\text { techniques }\end{array}$ & $\begin{array}{l}\text { Contrast } \\
\text { Enhancement } \\
\text { and noise } \\
\text { removal }\end{array}$ \\
\hline 3 & $\begin{array}{l}\text { Contrast } \\
\text { Enhancement }\end{array}$ & $\begin{array}{l}\text { Subtracting the } \\
\text { estimated global } \\
\text { illumination } \\
\text { from the given } \\
\text { image }\end{array}$ & $\begin{array}{l}\text { Histogram } \\
\text { Equalization and } \\
\text { Adaptive Histogram } \\
\text { Equalization }\end{array}$ \\
\hline 4 & $\begin{array}{l}\text { Noise } \\
\text { Filter }\end{array}$ & $\begin{array}{l}\text { Gaussian } \\
\text { low-pass } \\
\text { filter }\end{array}$ & $\begin{array}{l}\text { Wienner, Median, } \\
\text { NRCustom filter } \\
\text { apply }\end{array}$ \\
\hline 5 & GUI Based & No & Yes \\
\hline
\end{tabular}

Table 1 Comparison between previous and proposed work

In this paper, a comparative study had been carried out on five earlier proposed techniques that based on different features see Table 2.

Table 2 comparison between existing gender recognition methods and proposed approach

\begin{tabular}{|c|c|c|c|c|}
\hline Reference & Feature & Dataset & $\begin{array}{c}\text { No. of } \\
\text { Sample }\end{array}$ & Accuracy \\
\hline $\begin{array}{c}\text { Wenhao Zhang } \\
\text { et.al.[15] }\end{array}$ & $\begin{array}{c}\text { Fisher } \\
\text { Vector } \\
\text { Encoding }\end{array}$ & LFW & 1762 & $92.50 \%$ \\
\hline $\begin{array}{c}\text { M. M. Rahman } \\
\text { et.al.[14] }\end{array}$ & NABP & LFW & 1300 & $92.74 \%$ \\
\hline $\begin{array}{c}\text { Caifeng Shan } \\
\text { et. al.[16] }\end{array}$ & $\begin{array}{c}\text { Boosted } \\
\text { LBP }\end{array}$ & LFW & 7443 & $94.81 \%$ \\
\hline
\end{tabular}




\begin{tabular}{|c|c|c|c|c|}
\hline $\begin{array}{c}\text { Ramin } \\
\text { Azarmehr } \\
\text { et.al.[13] }\end{array}$ & MSLBP & FERET & 1762 & $95.19 \%$ \\
\hline $\begin{array}{c}\text { Miss.P.Sasikala } \\
\text { et.al. [17] }\end{array}$ & $\begin{array}{c}\text { Skin } \\
\text { color } \\
\text { model }\end{array}$ & $\begin{array}{c}\text { Web and } \\
\text { BioID } \\
\text { database }\end{array}$ & 502 & $89 \%$ \\
\hline Proposed Work & $\begin{array}{c}\text { Feature } \\
\text { Vector \& } \\
\text { Color } \\
\text { moment } \\
\text { with } \\
\text { AHE }\end{array}$ & $\begin{array}{c}\text { Web } \\
\text { Database }\end{array}$ & $\begin{array}{c}500 \\
\text { (Normal) }\end{array}$ & $98.16 \%$ \\
\hline
\end{tabular}

The Complete result of this comparison study carried out in tabular form as see the Table 5.3 and corresponding graph see the Figure 16

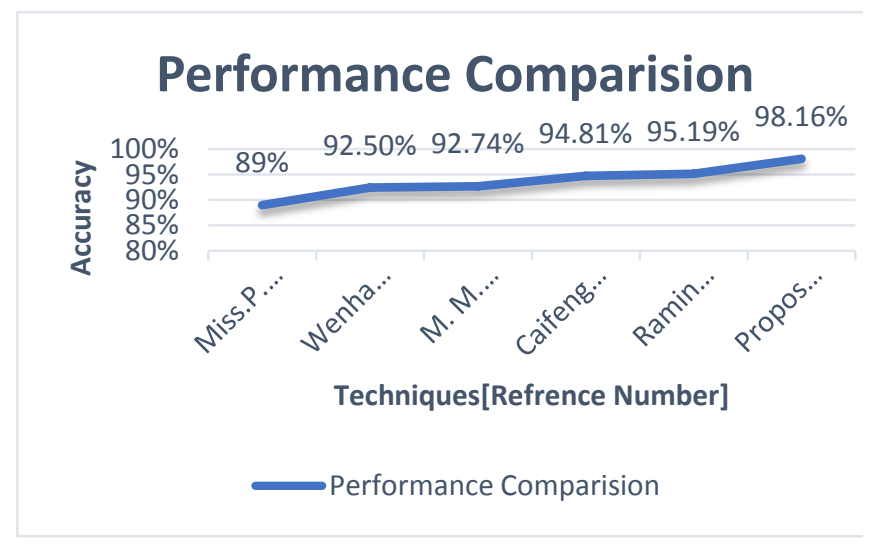

Fig. 16 Accuracy comparison with proposed work

Table: 3 Accuracies in various situations

\begin{tabular}{|l|c|c|c|}
\hline \multirow{2}{*}{\multicolumn{1}{c|}{$\begin{array}{c}\text { Feature } \\
\text { Extraction }\end{array}$}} & \multicolumn{3}{|c|}{ Accuracy (\%) } \\
\cline { 2 - 4 } & Normal & Dim & Dark \\
\hline $\begin{array}{l}\text { Proposed method } \\
\text { without AHE + } \\
\text { NRCustom }\end{array}$ & $94.71 \%$ & $92.85 \%$ & $88.09 \%$ \\
\hline $\begin{array}{l}\text { Proposed Method } \\
\text { with AHE + } \\
\text { NRCustom }\end{array}$ & $98.16 \%$ & $93.69 \%$ & $89.32 \%$ \\
\hline
\end{tabular}

Based on the above results, it can be observed that the proposed image enhancement algorithm performs much better than histogram equalization and contrast adjustment in terms of improving the face detection rates of difficult face image containing dark faces with bright background. For normal face images, our method also give better results. However, our method still performs better than existing method. Adaptive Histogram equalization also helps to improve the performance of the face detection and gender recognition algorithm. See Figure 17 and presented in Table 3. The proposed image enhancement algorithm produces enhanced image leading to the highest detection rates under almost all conditions.

\section{Accuracy comparision in different situations}

Normal Images

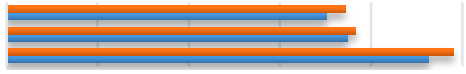

0.00\% 20.00\%40.00\%60.00\%80.00\%00.00\%20.00\%

- Proposed Method with AHE + NRCustom

- Proposed method without AHE + NRCustom

Fig. 17 Result comparison graph

\section{CONCLUSION AND FUTURE WORK}

In this paper, we have find problems of the face processing field, face detection, gender recognition. We reviewed recent developments in these two fields and our survey demonstrated that many of the existing methods suffer from different lighting conditions. In this work, we have proposed an efficient method for face detection and gender recognition based on features of face image. To improve the recognition accuracy of proposed system, we encode minimal amount of spatial information in the index by extracting features like color, feature vectors. Adaptive Histogram Equalization helps to detect skin area information from dark image and filter that information by proposed filter (NRCustom). This paper demonstrates the shape as well as color based feature extraction. The performance of the system can be better evaluated by system performance graphs, with social (Normal/Dim/Dark/ shaded) web Image database.

\section{REFERENCES}

[1] Mahmoud Afifia,b, Abdelrahman Abdelhamed" AFIF4: Deep Gender recognition based on AdaBoost-based Fusion of Isolated Facial Features and Foggy Faces", Elsevier, November 21, 2017.

[2] P. Comon, "Independent component analysis, a new concept?," Signal Processing, vol. 36, no. 3, pp. 287-314, 1994

[3] Huaining Cheng et. Al," gender and ethnicity classificationfrom small subsets of human body measurements", AFRL-RH-WP-TP-2014-0014.

[4] A. R . Ardakany, M ember, IACSIT and A. M. Jou la," Gender Recognition Based on Edge Histogram", International Journal of Computer Theory and Engineering Vol. 4, No. 2, April 2012.

[5] MandaVema Reddy," Face and Facial Expression Detection Using Viola-Jones and PCA Algorithm", Intenation journal and magazine of engineering, technology, management and research, ISSN No. 2348-4845.

[6] Yingxiao Wu, Yan Zhuang,et.al," Human Gender recognition: A Review", IEEE SENSORS JOURNAL, VOL. X, NO. X, XXXXXXX 2015.

[7] B Prabhavathi, V Tanuja, V Madhu Viswanatham and M Rajashekhara Babu," A smart technique for attendance system to recognize faces through parallelism", IOP Conf. 
Series: Materials Science and Engineering 263 (2017) 042095 doi:10.1088/1757-899X/263/4/042095.

[8] Eunsung L., Sangjin K., Wonseok K., Doochun S., and Joonki P. January 2014. Contrast Enhancement Using Dominant Brightness Level Analysis and Adaptive Intensity Transformation for Remote Sensing Images IEEE Geoscience and Remote Sensing Letters. Vol.10, No.1.

[9] W. Gao, B. Cao, S. Shan, D. Zhou, X. Zhang, and D. Zhao, "The CAS-PEAL largescale Chinese face database and baseline evaluations," Technical Report No. JDLTR_04_FR_001, Joint Research \& Development Laboratory, CAS, 2004.

[10] Arun Kumar Nagdeve, Somesh Kumar Dewangan,' automated facial feature points extraction", International Journal of Computer and Electronics Research [Volume 1, Issue 3, October 2012.

[11] A. Hadid, M. Pietikainen, and T. Ahonen, "A discriminative feature space for detecting and recognizing faces," in IEEE Computer Society Conference on Computer Vision and Pattern Recognition (CVPR), 2004, pp. II-797-II-804, vol. 2.

[12] Yujie Dong, Damon L.Woodard,"Eyebrow Shape-Based Features for biometric recognition and gender classification”, 978-1-4577-1359-0/11/\$26.00 @2011 IEEE.
[13] A. Ross, K. Nandakumar, and A. K. Jain, "Introduction to Multibiometrics," Handbook of Biometrics, A. Jain, P. Flynn and A. Ross, eds., pp. 271-292: Springer US, 2008.

[14] Ramin Azarmehr, Robert Laganiere, Won-Sook Lee, Christina $\mathrm{Xu}$, and Daniel Laroche. Real-time embedded age and gender recognition in unconstrained video. In Proceedings of the IEEE Conference on Computer Vision and Pattern Recognition Workshops, pages 57-65, 2015.

[15] M. M. Rahman, S. Rahman, M. Kamal, M. AbdullahAlWadud, E. K. Dey, and M. Shoyaib. Noise adaptive binary pattern for face image analysis. In 2015 18th International Conference on Computer and Information Technology (ICCIT), pages 390-395, 2015.

[16] Wenhao Zhang, Melvyn L Smith, Lyndon N Smith, and Abdul Farooq, "Gender recognition from facial images: two or three dimensions" JOSA A, 33:333-344, 2016.

[17] Caifeng Shan. Learning local binary patterns for gender recognition on real-world face images. Pattern Recognition Letters, 33:431-437, 2012.

$I{ }^{\prime T} A^{T M}$ : www.ijcaonline.org 